\title{
Comparing quantitative immunohistochemical markers of angiogenesis to subharmonic contrast imaging
}

\author{
Aditi Gupta $^{1,2}$, Mark A. Forsberg ${ }^{3}$, Kelly Dulin ${ }^{4}$, Samantha Jaffe ${ }^{4}$, Jaydev K. Dave ${ }^{1}$, \\ Valgerdur G. Halldorsdottir ${ }^{1,2}$, Andrew Marshall ${ }^{1,2}$, Anya I. Forsberg ${ }^{5}$, John Eisenbrey ${ }^{1}$, \\ Priscilla Machado ${ }^{1}$, Traci B. Fox ${ }^{1,6}$, Ji-Bin Liu ${ }^{1}$, Flemming Forsberg ${ }^{1}$ \\ ${ }^{1}$ Department of Radiology, Thomas Jefferson University, Philadelphia, PA 19107, USA \\ ${ }^{2}$ School of Biomedical Engineering, Sciences and Health Systems, Drexel University, \\ Philadelphia, PA 19104, USA \\ ${ }^{3}$ Yale University, CT 06520, USA \\ ${ }^{4}$ University of Pittsburgh, PA 15260, USA \\ ${ }^{5}$ Plymouth-Whitemarsh High School, Plymouth Meeting, PA 19462, USA \\ ${ }^{6}$ Department of Radiologic Sciences, College of Health Professions, Thomas Jefferson University, \\ Philadelphia, PA 19107, USA
}

\section{Abstract \\ Objective: Different methods for obtaining tumor neovascularity parameters based on immunohistochemical markers were compared to contrast-enhanced subharmonic ultrasound imaging (SHI).}

Methods: Eighty-five athymic, nude, female rats were implanted with $5 \times 10^{6}$ breast cancer cells (MDA-MB-231) in the mammary fat pad. The contrast agent Definity (Lantheus Medical Imaging, N Billerica, MA) was injected and SHI performed using a modified Sonix RP scanner (Analogic Ultrasound, Richmond, Canada) with a L9-4 linear array (transmitting/receiving frequencies $8 / 4 \mathrm{MHz}$ ). Afterwards, specimens were stained for endothelial cells (CD31), vascular endothelial growth factor (VEGF), and cyclooxygenase-2 (COX-2). Tumor neovascularity was assessed in 4 different ways using a histomorphometry system (100x magnification 1) over the entire tumor 2) in small sub-regions of interest (ROIs), 3) in the tumor periphery and centrally and 4) in 3 regions of maximum marker expression (so called hotspots). Results from specimens and from SHI were compared using linear regression.

Results: Fifty-four rats (64\%) exhibited tumor growth and 38 were successfully imaged. SHI depicted the tortuous morphology of tumor neovessels and delineated small areas of necrosis. The immunohistochemical markers did not correlate with SHI measures over the entire tumor area or over small sub-ROIs ( $>0.18)$. However, when the specimens were subdivided into a central and a peripheral region, COX-2 and VEGF correlated with SHI in the periphery $(r=-0.42 ; p=0.005$ and $\mathrm{r}=-0.32 ; \mathrm{p}=0.049$, respectively).

Correspondence: Flemming Forsberg, PhD, Thomas Jefferson University, Department of Radiology, 137 South $10^{\text {th }}$ St., 7 Main, Suite 763H, Philadelphia, PA 19107, USA, Tel: 215-955-4870, Fax: 215-955-8549, flemming.forsberg@ jefferson.edu. 
Conclusions: When comparing quantitative contrast measures of tumor neovascularity to immunohistochemical markers of angiogenesis in xenograft models ROIs corresponding to the biologically active region should be used to account for tumor heterogeneity.

\section{Keywords}

Subharmonic imaging; breast cancer; murine xenografts; immunohistochemistry

\section{Introduction}

Angiogenesis is the growth of new blood vessels, and is a fundamental physiological process for reproduction, development and wound repair $(1,2)$. Under normal circumstances, both the pro-angiogenic and anti-angiogenic factors of normal cells strike a perfect balance for controlled angiogenesis. Abnormal rapid proliferation of blood vessels (so called pathologic angiogenesis) plays an essential role in the growth of tumors and the development of metastases (1). The primary stimulus for new vessel formation is presumed to be hypoxia in the central region of the tumor (3). Pathologic angiogenesis provides a pathway for cancer cells to spread via the vascular and the lymphatic systems (1-5).

During angiogenesis tumors either secrete angiogenic substances directly, or activate and release angiogenic compounds stored within the extra-cellular matrix. Vascular endothelial growth factor (VEGF) promotes the formation of immature vessels, and is regarded as one of the most important pro-angiogenic factors of tumor angiogenesis $(6,7)$.

Cyclooxygenase-2 (COX-2), which is normally absent from cells, is expressed rapidly and induced transiently in response to tumor promoters, growth factors or cytokines (7). It is, thus, a positive regulator of angiogenesis. Platelet endothelial cell adhesion molecule (PECAM or CD31) is a potent endothelial cell marker, since its expression is restricted to vascular system platelets and endothelium $(2,7)$.

Ultrasound imaging has great potential in providing clinically relevant information related to angiogenesis by measuring tumor flow in real time and, when combined with gas microbubbles to perform contrast-enhanced ultrasound imaging, results improve even further $(8,9)$. Ultrasound contrast agents, made up of gas-filled and shell stabilized microbubbles less than $8 \mu \mathrm{m}$ in diameter, increase the signal-to-noise ratio by up to $25 \mathrm{~dB}(8)$. At higher acoustic pressures (>0.5 MPa) contrast microbubbles show nonlinear behavior that can be used in contrast-specific imaging modes $(8,9)$. When the second harmonic frequency component from the backscattered, nonlinear echoes is used to improve contrast visualization, it is known as harmonic imaging (HI), which is widely available on commercial ultrasound scanners. However, HI suffers from reduced blood-to-tissue contrast resulting from second harmonic generation and accumulation in tissue. Hence, subharmonic imaging (SHI), transmitting at the fundamental frequency $\left(\mathrm{f}_{0}\right)$ and receiving at the subharmonic $\left(\mathrm{f}_{0} / 2\right)$, becomes an attractive alternative contrast-specific imaging mode (1017). Subharmonic imaging has been shown to be more sensitive than HI for depicting angiogenesis, because tissue suppression and subharmonic signal strength is more important than the reduction in axial resolution in this application $(13,14)$. 
Tumors vary markedly over their surface volume in terms of morphological appearance and exhibit considerable variation in microvasculature structure and function (18). It is important to take this fact into consideration, when obtaining quantitative pathological parameters from tumors to use as reference standard for imaging studies. Recent studies have shown that assessment of various parameters of tumors yield superior results when the surface area/ volume is characterized into different regions of interest (ROIs) as compared to assessment over the whole tumor $(19,20)$. There is, however, no standard for how to analyze specimen markers and compare them to imaging parameters. Hence, we compared different methods for obtaining tumor neovascularity parameters based on immunohistochemical markers to contrast-enhanced SHI.

\section{Material and Methods}

Tumor model

Human breast adenocarcinoma cells (MDA-MB-231) were purchased from ATTC

(Manassas, VA) and $5 \times 10^{6}$ cells were mixed with matrigel (21) before being subcutaneously injected into the right mammary fat pads of 85 athymic, immunodeficient nude rats (RNU rats; Charles River Laboratories, Fredrick, MD). Out of the 85 rats, $64 \%$ (54 rats) showed tumor growth. Tumor growth was monitored twice per week over a period of 3 weeks and tumors that attained a size of at least $5 \times 5 \times 5 \mathrm{~mm}^{3}$ were selected for ultrasound scanning on the $21^{\text {st }}$ day, while the remaining were allowed to grow further. Similarly, tumors were selected for scanning on the $24^{\text {th }}$ and the $28^{\text {th }}$ day. Rats that did not show tumor growth beyond $5 \times 5 \times 5 \mathrm{~mm}^{3}$ prior to the $28^{\text {th }}$ day were euthanized. All the animal studies were carried out in an ethical fashion under the supervision of a veterinarian and were approved by the Institutional Animal Care and Use Committee of Thomas Jefferson University.

\section{Ultrasound data acquisition}

Prior to scanning, the rats were anesthetized using established techniques, and a lateral tail vein catheterized using a 24 gauge needle. The ultrasound contrast agent Definity (Lantheus Medical Imaging, N Billerica, MA) was injected (dose: $180 \mu \mathrm{l} / \mathrm{kg}$ ) followed by a $0.2 \mathrm{ml}$ saline flush. The United States' Food and Drug Administration has approved Definity, which consists of phospholipid shelled, octaflouropropane microbubbles 1.1 to $3.3 \mu \mathrm{m}$ in diameter (22), for use in echocardiography. A total of three injections of contrast were administered for each tumor. The time between injections was 3 to 5 minutes to allow the agent to clear the blood pool.

A Sonix RP scanner (Analogic Ultrasound, Richmond, BC, Canada) with a L9-4 linear array transducer was modified to operate in pulse-inversion, grayscale SHI mode transmitting at 8 $\mathrm{MHz}$ and receiving at $4 \mathrm{MHz}$ using an acoustic output power of $-10 \mathrm{~dB}$ (approximately 760 $\mathrm{kPa}$ peak-to-peak in situ pressure or an MI of about 0.13). The parameters used for image acquisition were selected based on our prior experiences (23) and are summarized in Table 1. 
Coupling gel was liberally applied on the tumor to create a standoff pad to minimize pressure on the tumor from the ensuing placement of the transducer. The tumors of all rats were initially imaged in conventional grayscale mode and their width, depth and height were measured. The sonographer then imaged the largest transverse cross section of each individual tumor, and this imaging plane was held constant for the entire experiment. Digital clips of each contrast injection were captured for off-line analysis.

\section{Pathology data acquisition}

After the ultrasound scans were completed, the animals were euthanized using standard techniques. Tumors were then surgically removed and scanned ex vivo to identify the imaging plane that was studied in vivo. Following fixation, specimens were dissected in 10 $\mu \mathrm{m}$ slices corresponding to the imaging plane and stained immunohistochemically against VEGF, CD31 and COX-2. A monoclonal antibody against PECAM (anti CD31; Dako Corporation, Carpinteria, CA), a monoclonal antibody against VEGF (Oncogene Research Products, San Diego, CA) and a polyclonal antibody against COX-2 (Santa Cruz Biotechnology, Santa Cruz, CA) were utilized for staining. Finally, the stained sections were mounted onto glass slides.

\section{Data analysis}

A semi-automated histomorphometry system was used to obtain and analyze digital images of each histologic slide (24-26). A DXC-970MD color CCD camera (Sony Corporation, Tokyo, Japan) was used in conjunction with a Labophot-2 microscope (Nikon, Melville, NJ) set to 10x ocular magnification with a 10x objective (total magnification 100x). Between 20 and 130 images were acquired to encompass the entire tumor. This was accomplished using a motorized stage controlled by ImagePro Plus software (Media Cybernetics, Silver Spring, $\mathrm{MD}$ ). This software was then used to obtain red-green-blue (RGB) images of the entire specimen. In cases where the tumor occupied less than $25 \%$ of the image, it was outlined manually. Images obtained after subtraction were subjected to $3 \times 3$ open and sharpen filters for enhancement (24). The RGB channels were then used to count the number of stained pixels $\left(\mathrm{s}_{\mathrm{i}}\right)$ in each image and the ratio to the total number of pixels $\left(\mathrm{s}_{\mathrm{i}}+\mathrm{x}_{\mathrm{i}}\right)$ representing the percent stained area or relative expression (RE) was calculated according to (24-26):

$$
R E=\frac{\Sigma s_{i}}{\left(\Sigma s_{i}+x_{i}\right)}
$$

The RE obtained for all three immunohistochemical markers (i.e., VEGF, CD31 and COX-2) were then organized into marker specific pathological maps.

Since the nature of tumor vasculature is heterogeneous (1), tumor neovascularity was assessed in 4 different ways as follows (26):

1. Over the entire tumor: In this case, the average RE over the entire pathological map of the tumor was calculated.

2. In small sub-ROIs: In this case, the pathological map of the tumor was divided into smaller ROIs ( $3-4 \mathrm{~mm}$ ) based on the size of the tumor, and the average RE 
for each ROI was calculated. This was done to account for the heterogeneity of the tumor and examine different regions of the tumor separately (25).

3. In the tumor periphery (within $2 \mathrm{~mm}$ of the margin) and centrally: In this case, the pathological map of the tumor was divided into the center and the periphery, and average RE was calculated for center, and periphery. This was done to better account for the fact that tumors are necrotic in the center, and vascular in the periphery (1).

4. In regions of high $\%$ stained area in the tumor (hotspots): In this case, 3 regions of maximum \% stained area were considered from each pathological map of the tumor. This was done to focus on areas of high vascularity within the tumor (i.e., more biologically active areas) and compare them with corresponding regions of the SHI images.

The average \% stained area (or RE) obtained for different ROIs from each of the above mentioned cases was then compared to the corresponding ROIs in the SHI ultrasound images.

In order to study the heterogeneous and tortuous morphology of tumor angiogenesis in the SHI images, the maximum intensity projection (MIP) technique was implemented in MATLAB (R2014a, The Mathworks Inc., Natick, MA). MIP involves the projection of pixels with maximum intensity in the plane of projection. Once the MIP images were obtained, the ROI in each case was outlined manually with the help of a radiologist, corresponding to the ROIs marked on the pathological maps of tumor specimens. The algorithm uses Otsu's segmentation technique, which is aimed at finding the optimal value for a global threshold. The algorithm thus, calculated the fractional vascularity (FV) of the selected ROI by classifying the SHI image pixels into tumor vascular pixels or tumor background pixels and then taking the ratio of the two as an expression of the relative tumor vascularity. This was followed by the display of the composite image, which showed the tumor vasculature architecture for the selected ROI.

In case of the assessment of tumor neovascularity using the hotspots method, the ROI was selected differently by a modified version of the algorithm. In this case the ROI was a fixed size rectangle ( $45 \times 30$ pixels), which could be dragged anywhere across the tumor image and placed on the respective region under consideration (decided from the 3 highest RE regions in the pathological maps).

\section{Statistical analysis}

The RE values from the tumor specimens (for each of the three immunohistochemical markers) were compared to FV results obtained from SHI (averaged for each tumor over the 3 injections) using linear regression analysis. Paired t-tests were used to compare all 4 variables (SHI FV as well as the expression of the three immunohistochemical markers) in the tumor periphery versus the center. Correlation coefficients and statistically significant differences (assuming $a=0.05$ and $95 \%$ confidence intervals) were obtained for all 4 cases of assessment using Stata 11 (Stata Corp, College Station, TX). 


\section{Results}

Out of the 54 rats that showed some tumor growth, 38 were successfully imaged using SHI. The other 16 animals either had very limited tumor growth $(\mathrm{N}=12)$, or in 4 cases the Sonix RP scanner experienced technical difficulties and SHI data was not acquired. The tumors that grew in the 38 rats studied had an average diameter of $9.4 \mathrm{~mm} \pm 5.3 \mathrm{~mm}$. A representative SHI scan of a breast tumor demonstrates the tortuous morphology of tumor neovessels as well as areas of necrosis (Figure 1). Examples of the tumor specimens and maps (the latter stained for CD31) are presented in Figures 2 and 3.

For the whole tumor case, the linear regression analysis showed no significant correlation between tumor neovascularity assessed by SHI (i.e., FV) and any of the immunohistochemical markers ( $p>0.60$; Table 2). Likewise, when FV SHI from multiple sub-ROIs or the center of the tumor was compared to the percent area stained (i.e., RE) with CD31, COX-2 or VEGF there were no significant correlations ( $p>0.18$ ). Conversely, when ROIs corresponding to the tumor periphery were analyzed there was a strong correlation between tumor vascularity depicted by SHI and the RE obtained for COX-2 $(r=-0.42 ; \mathrm{p}=$ 0.005; Table 2) as well as for VEGF $(r=-0.32 ; \mathrm{p}=0.049$; Table 2). Scatter plots for those two cases are shown in Figure 4. There was no significant correlation obtained when comparing SHI and CD31 in the tumor periphery $(\mathrm{p}=0.21)$. This was also the case when SHI FV and CD31 or VEGF RE values were compared ( $p \geq 0.23$ ). While there was a notable correlation seen between SHI FV and COX-2 RE ( $r=0.25 ; p=0.007$; Table 2), this data set was dominated by a few large outliers and showed marked variability (data not shown for the sake of brevity).

When values from the center and the periphery of the tumors were compared SHI ( $\mathrm{p}<$ $0.001)$ and COX-2 ( $=0.030)$ showed significant differences (Figure 5); unlike REs obtained for VEGF and CD31 ( $p>0.18$ ). There is more flow (vascularity) observed with $\mathrm{SHI}$ in the periphery as expected. In the case of COX-2, although the difference between center and periphery is statistically significant, it is less convincing in a small sample size (cf., Figure 5).

\section{Discussion}

In this study, murine breast tumor FV was quantified from SHI images and compared to the RE of three angiogenic markers. The SHI images showed a negative correlation with the expression of COX-2 and VEGF (cf., Table 2; Figure 4) when the ROI considered was the periphery of the tumor, which is known to have increased biological activity and vascularity (as compared to the potentially necrotic center). Our group previously reported on reduced tumor vascularity observed with contrast enhanced ultrasound corresponding to increased expression of COX-2 in a melanoma xenograft model (7). It was speculated that the rapidly growing tumors must have shifted the distribution of their microvessels towards the periphery as they enlarged. Decreased tumor vascularity with increasing expression of VEGF has also been reported in different preclinical xenograft models $(19,20)$. Thus, contrast enhanced SHI appears to provide a non-invasive marker for angiogenesis in the MDA-MB-231 murine xenograft model. 
Results from this study also demonstrate that tumor heterogeneity is an important factor that should be considered, while performing any type of quantification involving tumor neovascularity (cf., Table 2). There are regions of tumors that will show higher vasculature formation than others, and these might also differ in morphological appearance and function (1-3). The negative correlation between the tumor vascularity obtained with SHI and the immunohistochemical markers of angiogenesis (in particular COX-2) suggests that there is decreased angiogenic marker expression in areas of increased vascularity (i.e., the tumor periphery). This observation is also supported by results of other breast cancer studies, demonstrating an inverse relationship between tumor volume and tumor microvessel density, and higher microvessel density at the tumor periphery than at the center (27).

It should be mentioned that while the entire tumor area was taken into consideration for the flow assessments conducted as in this study (cf., Figure 3), the entire tumor volume (i.e., 3D neovascularity) was not evaluated. Also, the elevation thickness of the SHI ultrasound pulse is much greater than the specimen slice thickness ( $\mathrm{mm}$ vs. $\mu \mathrm{m})$ indicating that even though great care was taken to align the imaging planes with the specimens an exact match cannot be achieved. Another scope for error exists in the manual marking of the ROIs on the SHI tumor images, which might not have completely matched the hotspots in the pathology specimens. Moreover, the tumor vascular endothelium was not labelled in vivo (e.g., with tomato lectin (28)), which may confound comparisons of SHI vascularity and ex vivo angiogenic marker expression. Finally, it is a statistical limitation of this study that multiple comparisons (i.e., 4) are being conducted on the same data set (i.e., the same tumors). A Bonferroni correction, which assigns the traditional $0.05 \mathrm{p}$-value divided by the number of comparisons (so $0.05 / 4=0.0125$ ) to be the $p$-value of significance, could be used to compensate (29). However, by controlling the group-wise error rate, each individual test is held to an unreasonably high standard increasing the likelihood that legitimately significant results will be discarded (30). Hence, we chose not to apply Bonferroni correction in this study.

In conclusion, contrast enhanced SHI holds potential as a diagnostic tool for monitoring angiogenesis of breast tumors; at least based on the results in this pre-clinical rat model. Most importantly, when comparing quantitative contrast measures of tumor neovascularity to immunohistochemical markers of angiogenesis in xenograft models ROIs corresponding to the biologically active region should be used to account for tumor heterogeneity and development.

\section{Acknowledgements}

This work was supported in part by U.S. Army Medical Research Material Command under W81XWH-08-1-0503, W81XWH-12-1-0066. Definity was supplied by Lantheus Medical Imaging, N. Billerica, MA. Part of this work was previously published in abstract and proceedings (16, 31-32).

Supported in part by U.S. Army Medical Research Material Command under W81XWH-08-1-0503, W81XWH-12-1-0066 and by Lantheus Medical Imaging, N. Billerica, MA, USA.

\section{References}

1. Li WW. Tumor angiogenesis: molecular pathology, therapeutic targeting, and imaging. Acad Radiol 2000; 10:800-811. 
2. Pircher A, Hilbe W, Heidegger I, et al. Biomarkers in tumor angiogenesis and anti-angiogenic therapy. Int J Mol Sci, 2011; 12:7077-7099. [PubMed: 22072937]

3. Jeswani T, Padhani AR. Imaging tumour angiogenesis. Cancer Imaging 2005; 5:131-138. [PubMed: 16321775]

4. Folkman J What is the evidence that tumors are angiogenesis dependent? J Natl Cancer Inst 1990; 82:4-6. [PubMed: 1688381]

5. Holmgren L, O’Reilly MS, Folkman J. Dormancy of micrometastases: balanced proliferation and apoptosis in the presence of angiogenesis suppression." Nat Med 1995; 1:149-153. [PubMed: 7585012]

6. Drevs J VEGF and angiogenesis: implications for breast cancer therapy. Eur J Cancer (Supplement) 2008; 6:7-13.

7. Forsberg F, Ro R, Potoczek M, et al. Assessment of angiogenesis: implications for ultrasound imaging. Ultrasonics 2004; 42:325-330. [PubMed: 15047306]

8. Eisenbrey JR, Forsberg F. Contrast-enhanced ultrasound for molecular imaging of angiogenesis. Eur J Nuc Med Mol Imaging 2010; 37:138-146.

9. Davidson BP, Lindner JR. Future applications of contrast echocardiography. Heart. 2012; 98:246253. [PubMed: 22199019]

10. Forsberg F, Shi WT, Goldberg BB. Subharmonic imaging of contrast agents. Ultrasonics 2000, 38:93-98. [PubMed: 10829636]

11. Forsberg F, Piccoli CW, Merton DA, Palazzo JJ, Hall AL. Breast lesions: imaging with contrastenhanced subharmonic US - initial experience. Radiology 2007, 244:718-726. [PubMed: 17690324]

12. Eisenbrey JR, Dave JK, Halldorsdottir VG, et al. Simultaneous grayscale and subharmonic ultrasound imaging on a modified commercial scanner. Ultrasonics, 2011; 51:890-897. [PubMed: 21621239]

13. Sridharan A, Eisenbrey JR, Machado P, et al. Quantitative analysis of vascular heterogeneity in breast lesions using contrast-enhanced three-dimensional harmonic and subharmonic ultrasound imaging. IEEE Trans Ultrason, Ferroelec Freq Control, 2015; 62:502-510.

14. Shankar PM, Krishna PD, Newhouse VL. Advantages of subharmonic over second harmonic backscatter for contrast-to-tissue echo enhancement. Ultrasound Med Biol 1998; 24:395-399. [PubMed: 9587994]

15. Chomas J, Dayton P, May D, Ferrara K. Nondestructive subharmonic imaging. IEEE Trans. Ultrason. Ferroelec. Freq. Contr, 2002; 49:883-892.

16. Goertz DE, Frijlink ME, Tempel D, et al. Subharmonic contrast intravascular ultrasound for vasa vasorum imaging. Ultrasound Med Biol 2007; 33:1859-1872. [PubMed: 17683850]

17. Faez T, Emmer M, Docter M, et al. Characterizing the subharmonic response of phospholipidcoated microbubbles for carotid imaging. Ultrasound Med Biol. 2011; 37:958-970. [PubMed: 21531498]

18. Garcia-Closas M, Hall P, Nevanlinna H, et al. Heterogeneity of breast cancer associations with five susceptibility loci by clinical and pathological characteristics. PLoS Genet 2008; 4:e1000054. [PubMed: 18437204]

19. Forsberg F, Ro RJ, Marshall A, et al. The antiangiogenic effects of a vascular endothelial growth factor decoy receptor can be monitored in vivo using contrast-enhanced ultrasound imaging. Mol Imaging. 2014; 13:1-9.

20. Eichten A, Adler AP, Cooper B, et al. Rapid decrease in tumor perfusion following VEGF blockade predicts long-term tumor growth inhibition in preclinical tumor models. Angiogenesis. 2013; 16:429-441. [PubMed: 23238831]

21. Lewis MT, Landua JD, Adams HC 3rd, Medina D. A mystery wrapped in an enigma: Matrigel enhancement of mammary cell growth and morphogenesis. J Mammary Gland Biol Neoplasia. 2012; 17:99-101. [PubMed: 22581302]

22. Miller AP, Nanda NC. Contrast echocardiography: new agents. Ultrasound Med Biol., 2004; 30:425-434. [PubMed: 15121243] 
23. Halldorsdottir VG, Dave JK, Eisenbrey JR, et al. Subharmonic aided pressure estimation for monitoring interstitial fluid pressure in tumours--in vitro and in vivo proof of concept. Ultrasonics. 2014; 54:1938-44. [PubMed: 24856899]

24. Forsberg F, Kuruvilla B, Pascua MB, et al. Comparing contrast-enhanced color flow imaging and pathological measures of breast lesion vascularity. Ultrasound Med Biol. 2008; 34:1365-1372. [PubMed: 18436369]

25. Forsberg F, Ro RJ, Fox TB, et al. Contrast enhanced maximum intensity projection ultrasound imaging for assessing angiogenesis in murine glioma and breast tumor models: A comparative study. Ultrasonics. 2011; 51:382-389. [PubMed: 21144542]

26. Gupta A, Forsberg MA, Dulin K, et al. Comparing subharmonic imaging and immunohistochemical markers of angiogenesis. Proc IEEE IUS, 2014;1156-1159.

27. Wapnir IL, Barnard N, Wartenberg D, Greco RS. The inverse relationship between microvessel counts and tumor volume in breast cancer. Breast J. 2001; 7:184-188. [PubMed: 11469933]

28. Mazzetti S, Frigerio S, Gelati M, et al. Lycopersicon esculentum lectin: an effective and versatile endothelial marker of normal and tumoral blood vessels in the central nervous system. Eur J Histochem 2004; 48:423-428. [PubMed: 15718209]

29. Bland JM, Altman DG. Multiple significance tests: the Bonferroni method. Br Med J 1995; 310:170. [PubMed: 7833759]

30. Perneger TV. What's wrong with Bonferroni adjustments. Br Med J 1998; 316:1236-1238. [PubMed: 9553006]

31. Gupta A, Dulin K, Jaffe S, et al. Quantifying tumor neovascularity with immunohistochemical markers compared to subharmonic US imaging (abstract). Prog RSNA 2014; abstract no PHS136.

32. Gupta A, Jaffe S, Forsberg MA, et al. On techniques for comparing contrast imaging and specimen markers of angiogenesis (abstract). J Ultrasound Med 2014; 33:S12. 


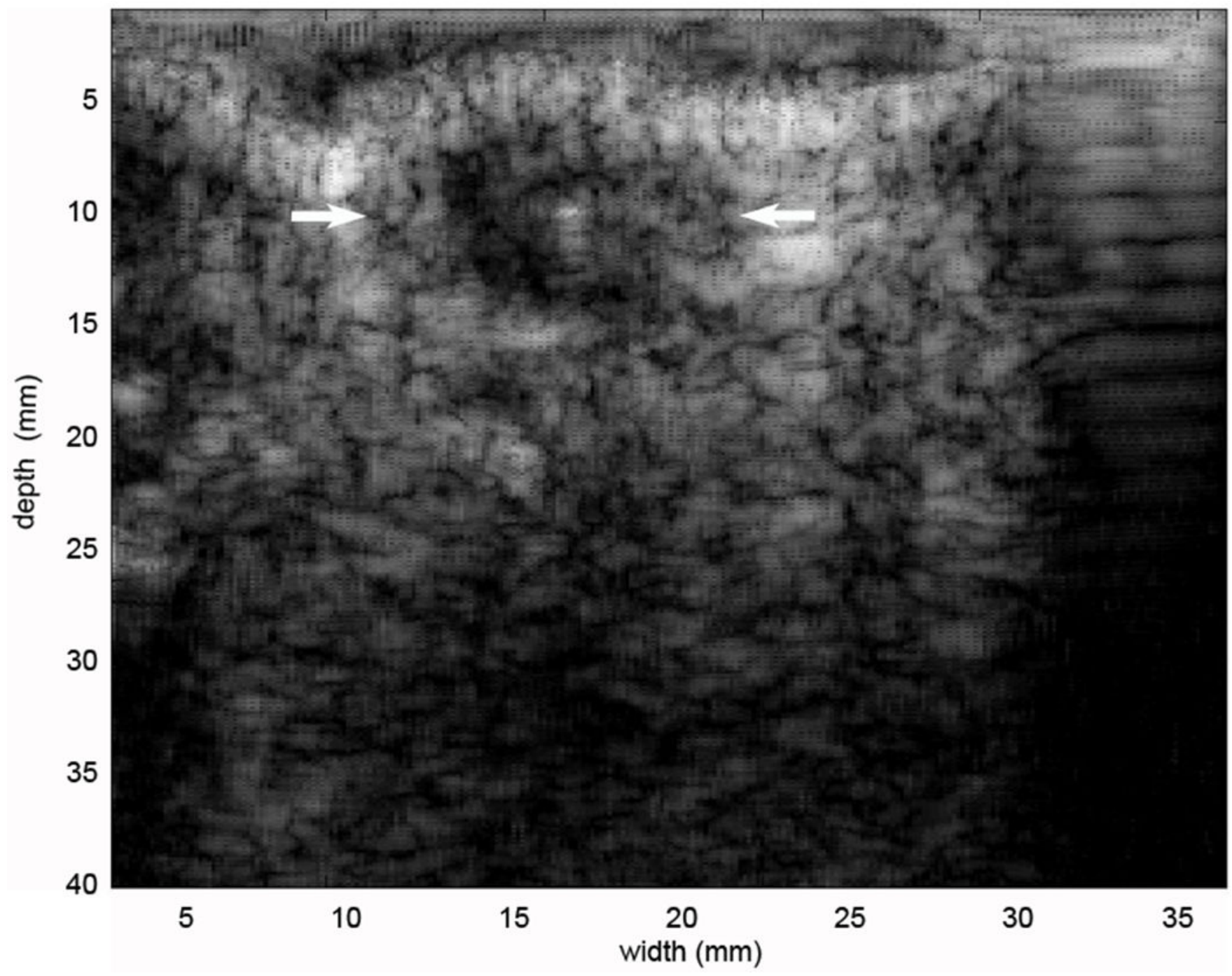

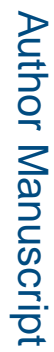




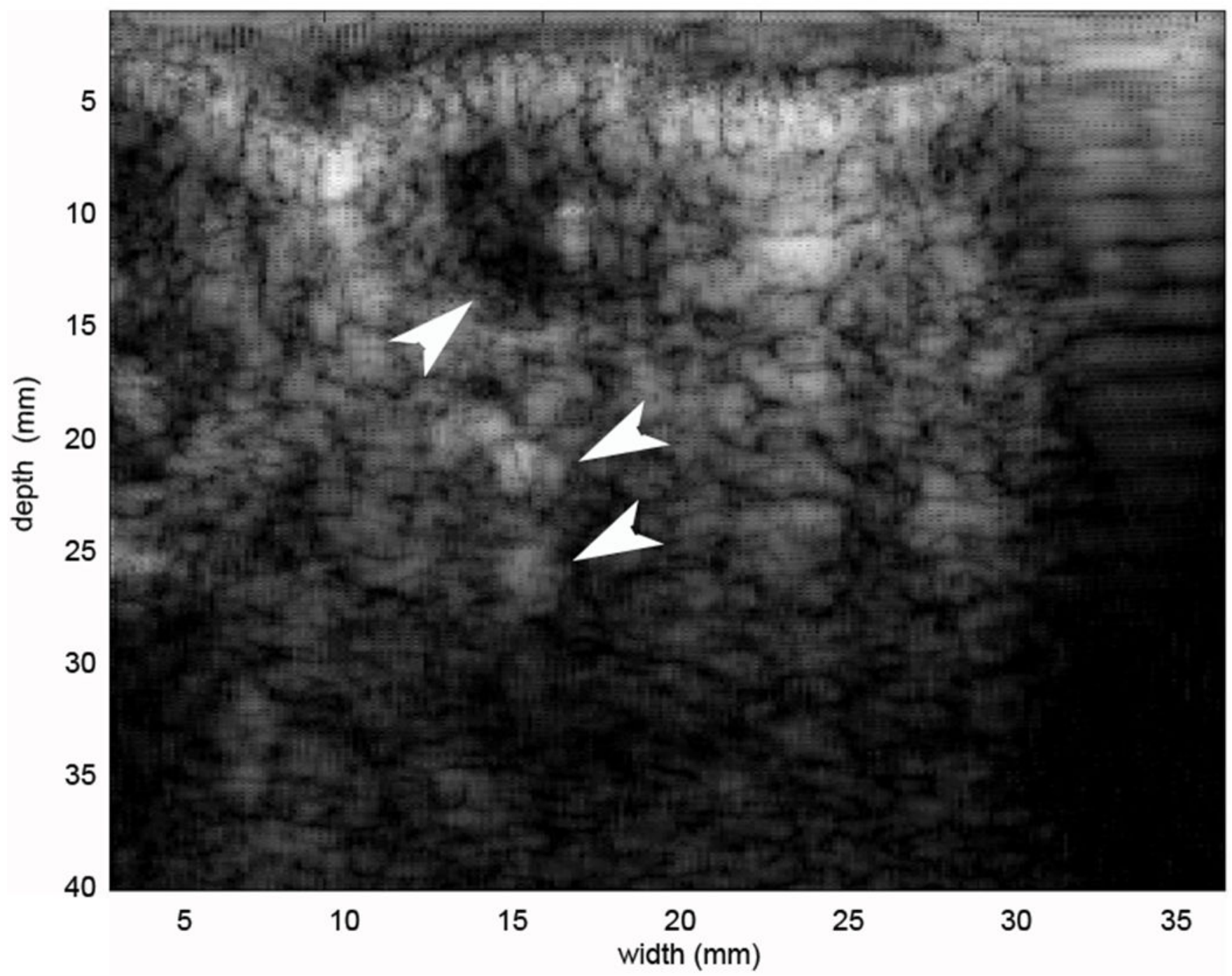

Figure 1.

A murine breast tumor (arrows) scanned in SHI mode before (a) and after (b) administration of Definity. Notice the larger vessel outside the tumor as well as the smaller vessels inside (arrow heads). The size of this tumor is $11 \times 9 \mathrm{~mm}$. 


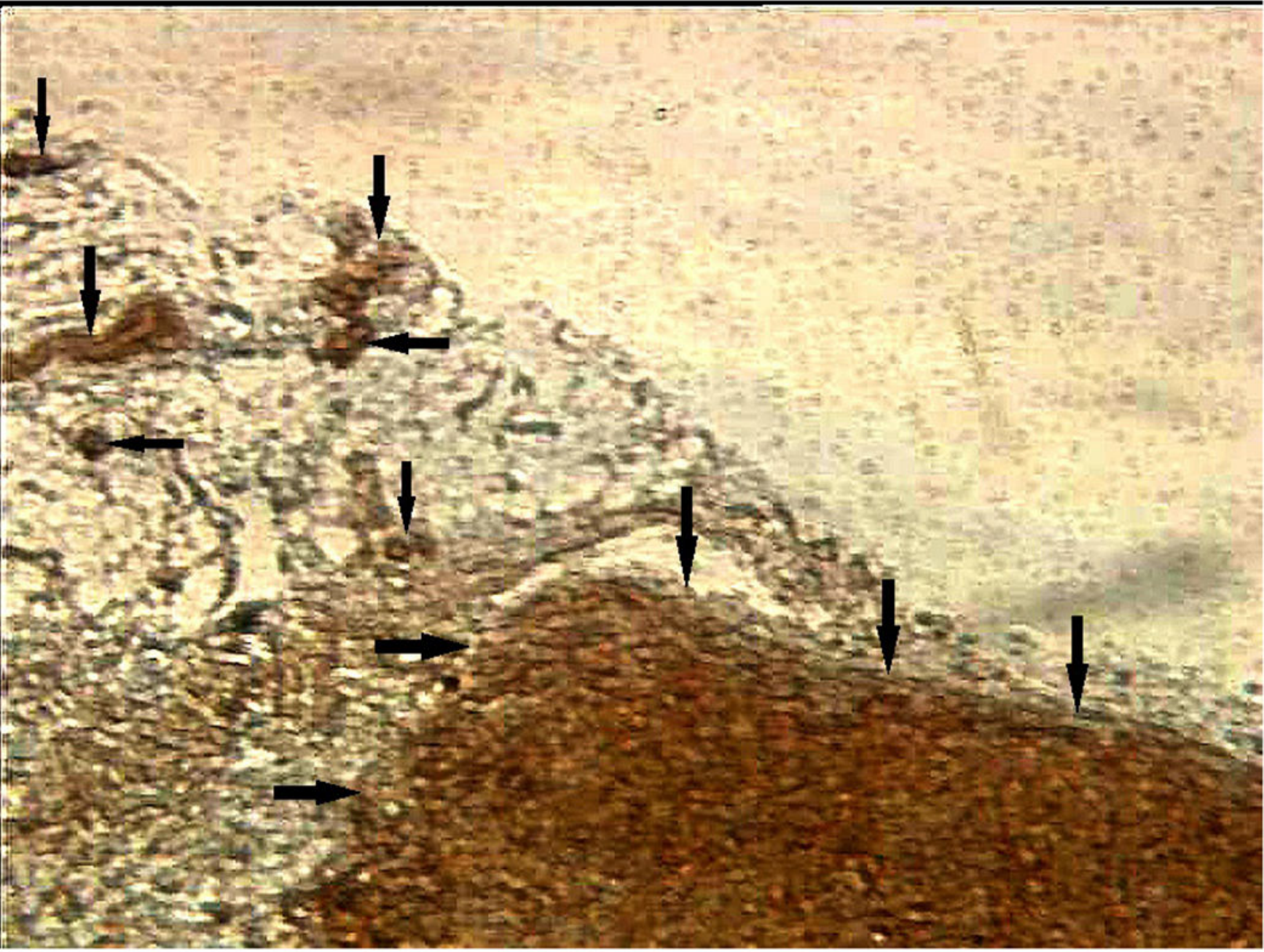

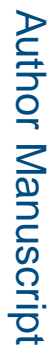




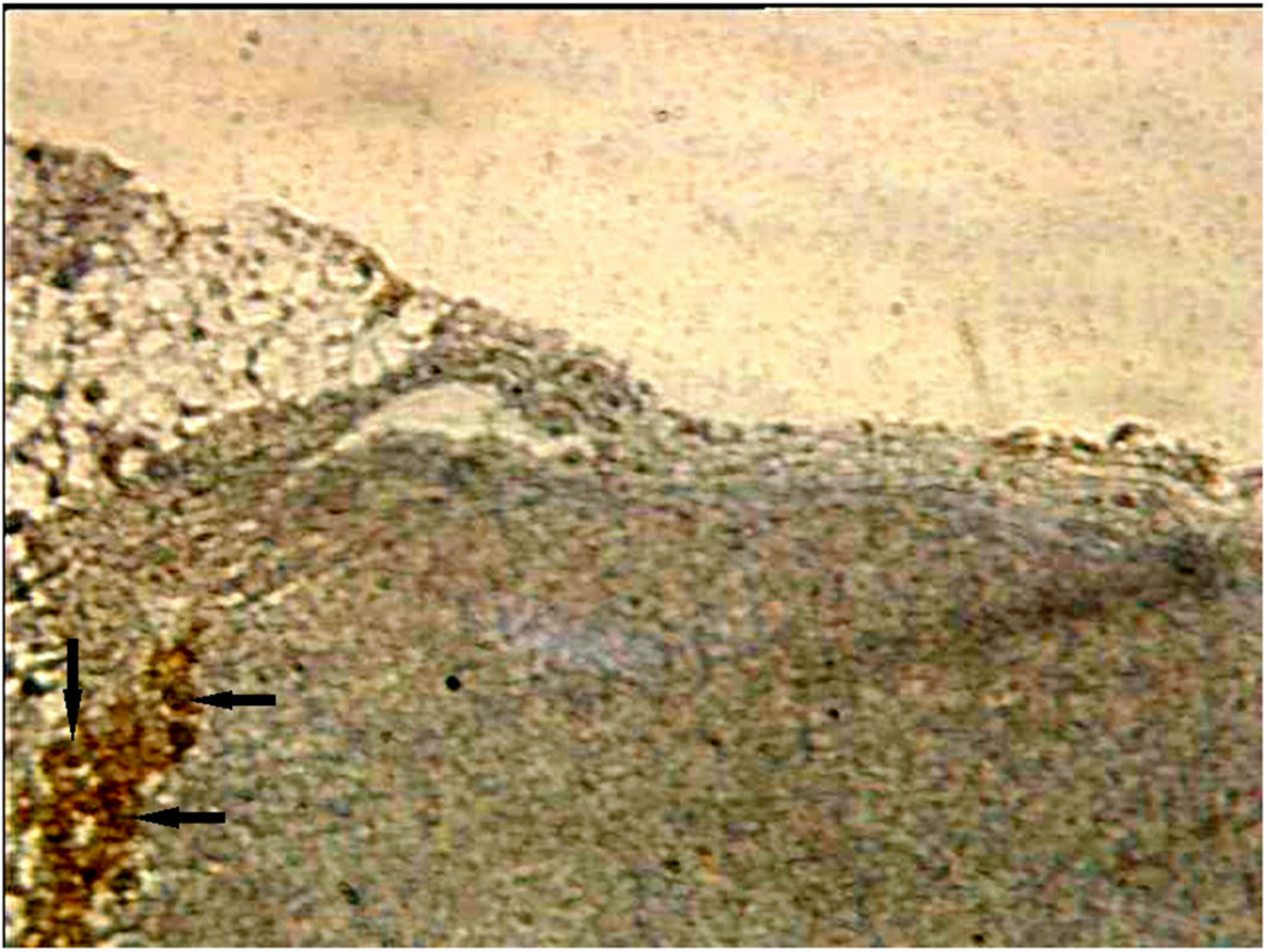

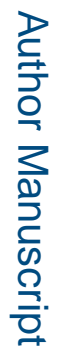




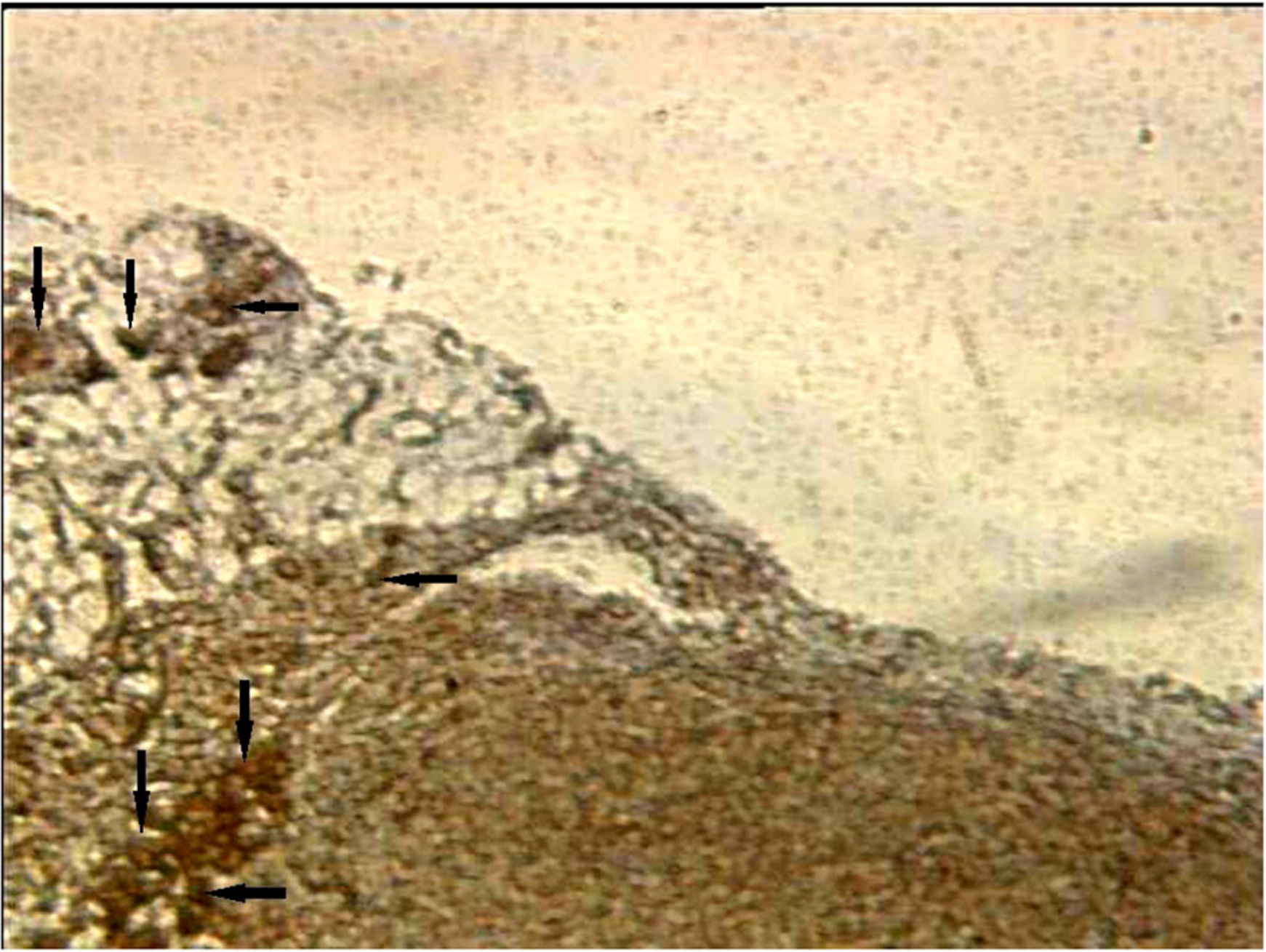

Figure 2:

Immunohistochemical staining (arrows) of tumor (from Figure 1) for (a) VEGF, (b) CD31 and (c) $\mathrm{COX}-2$. 


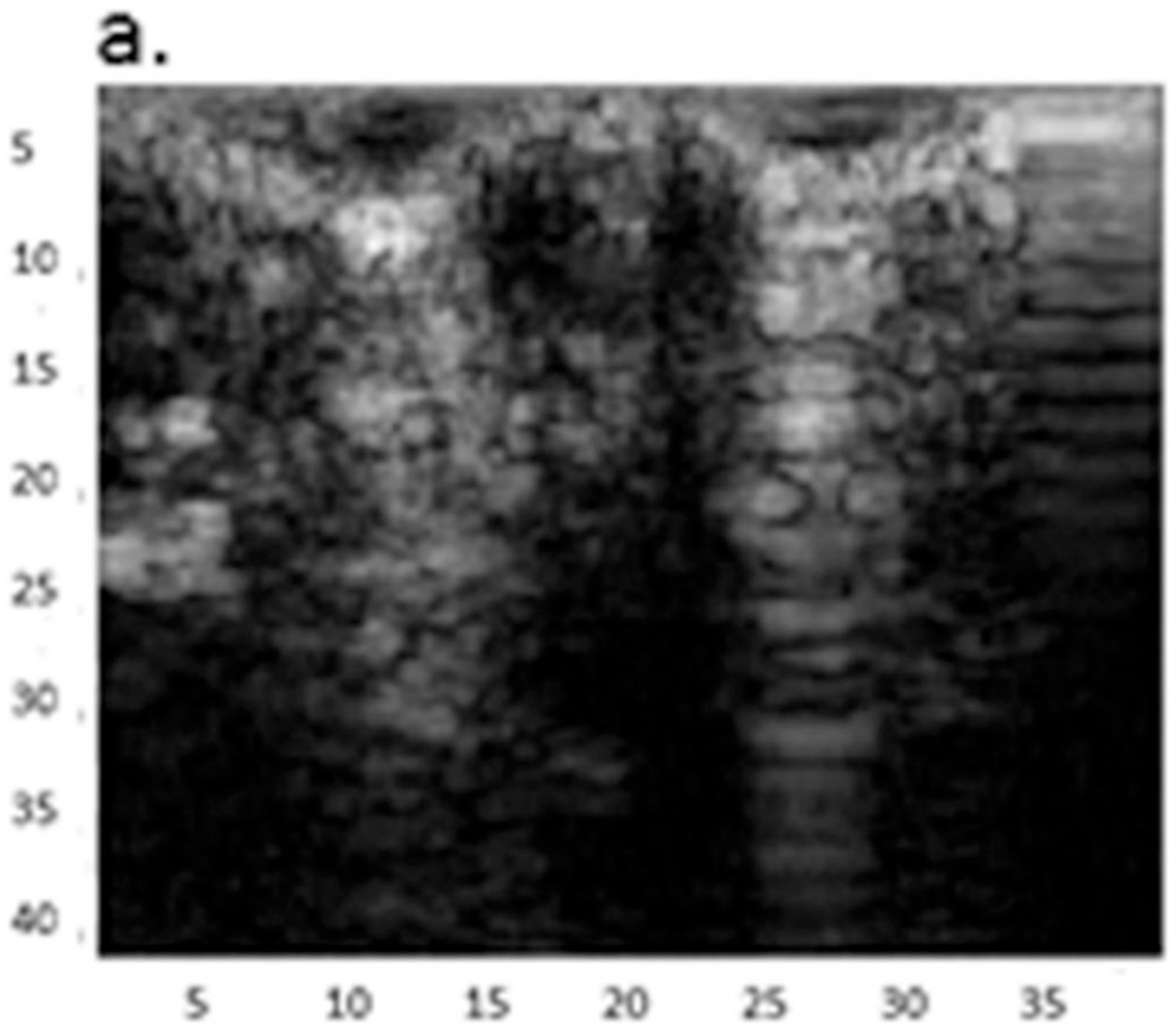




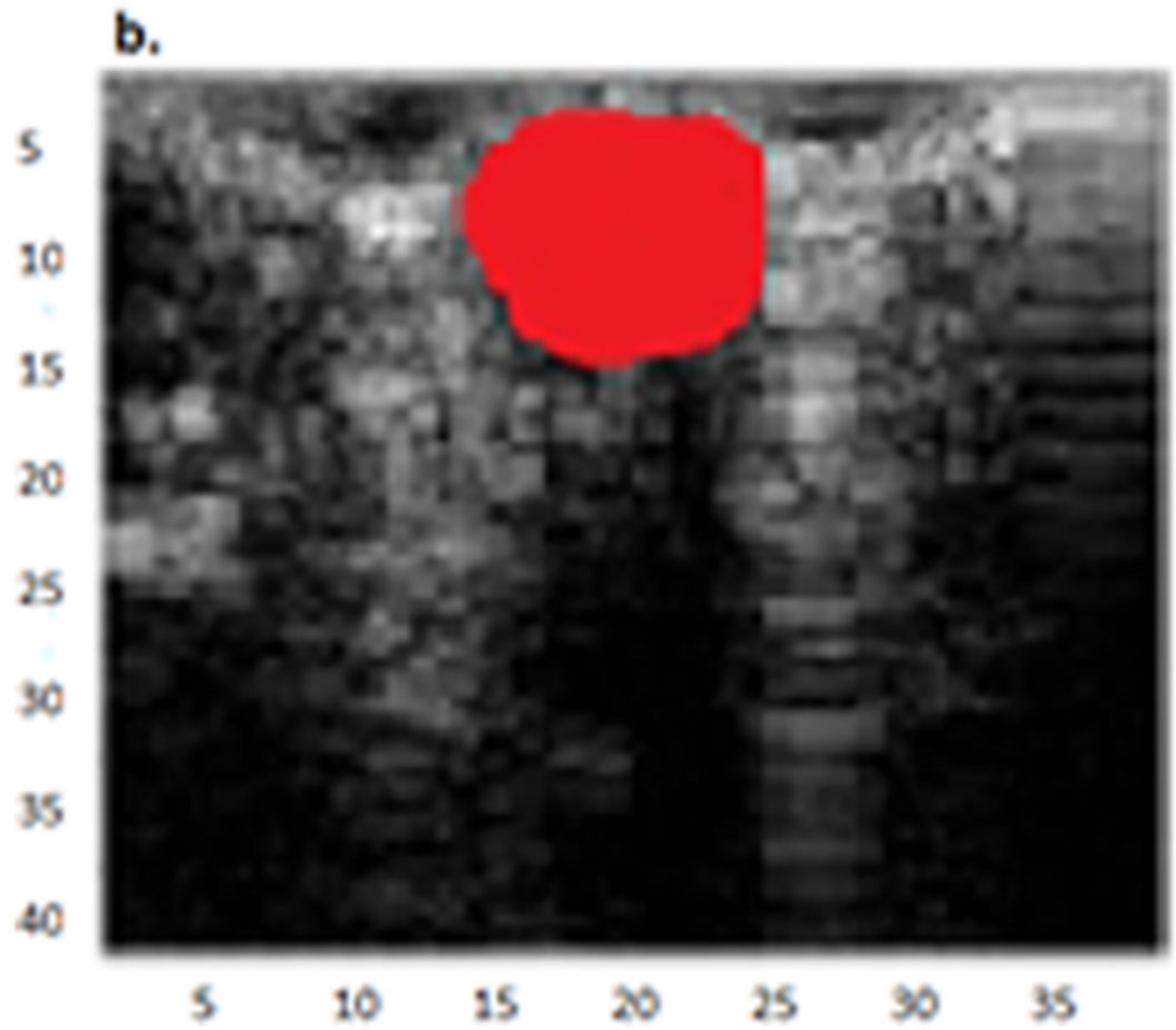

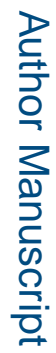




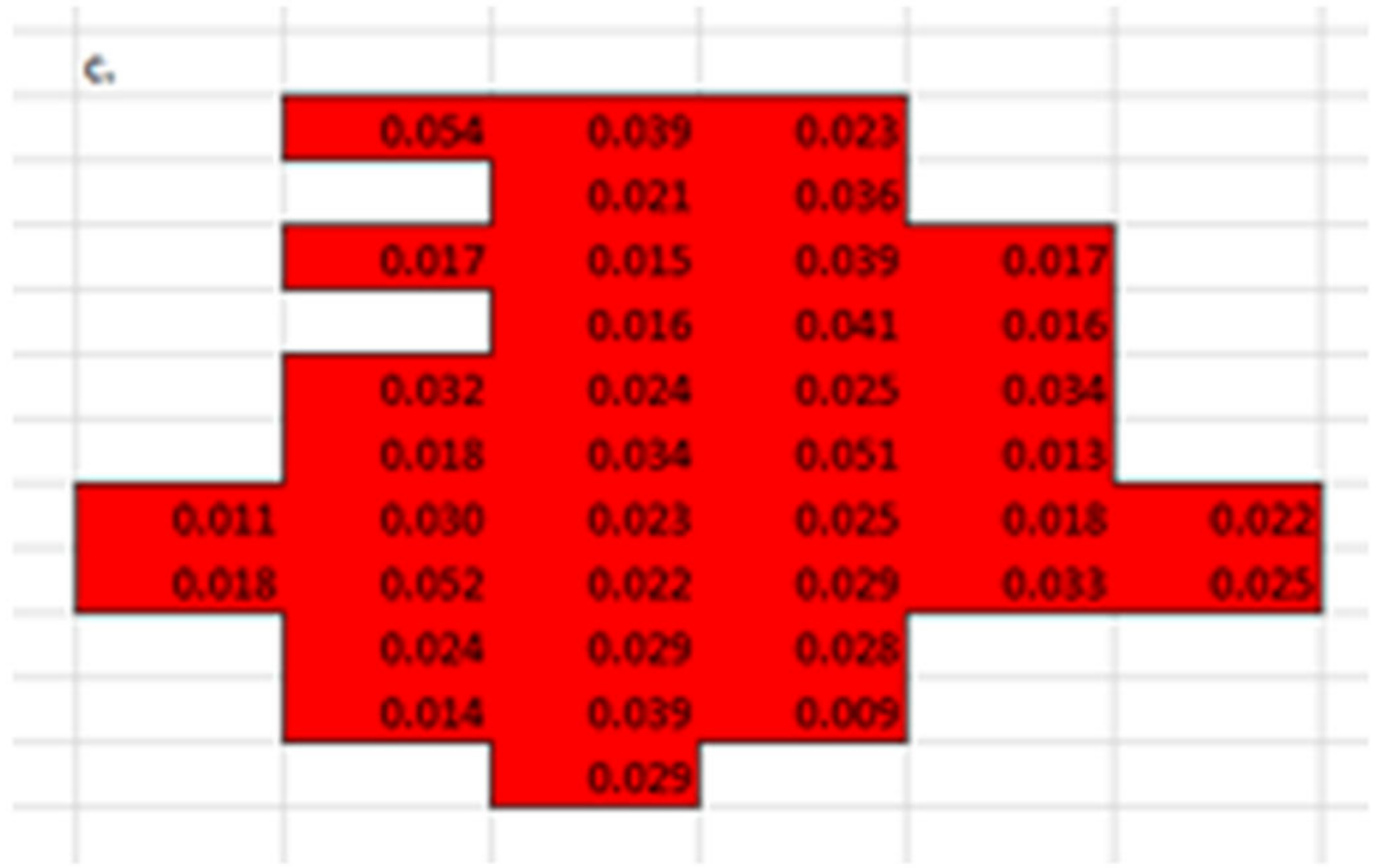




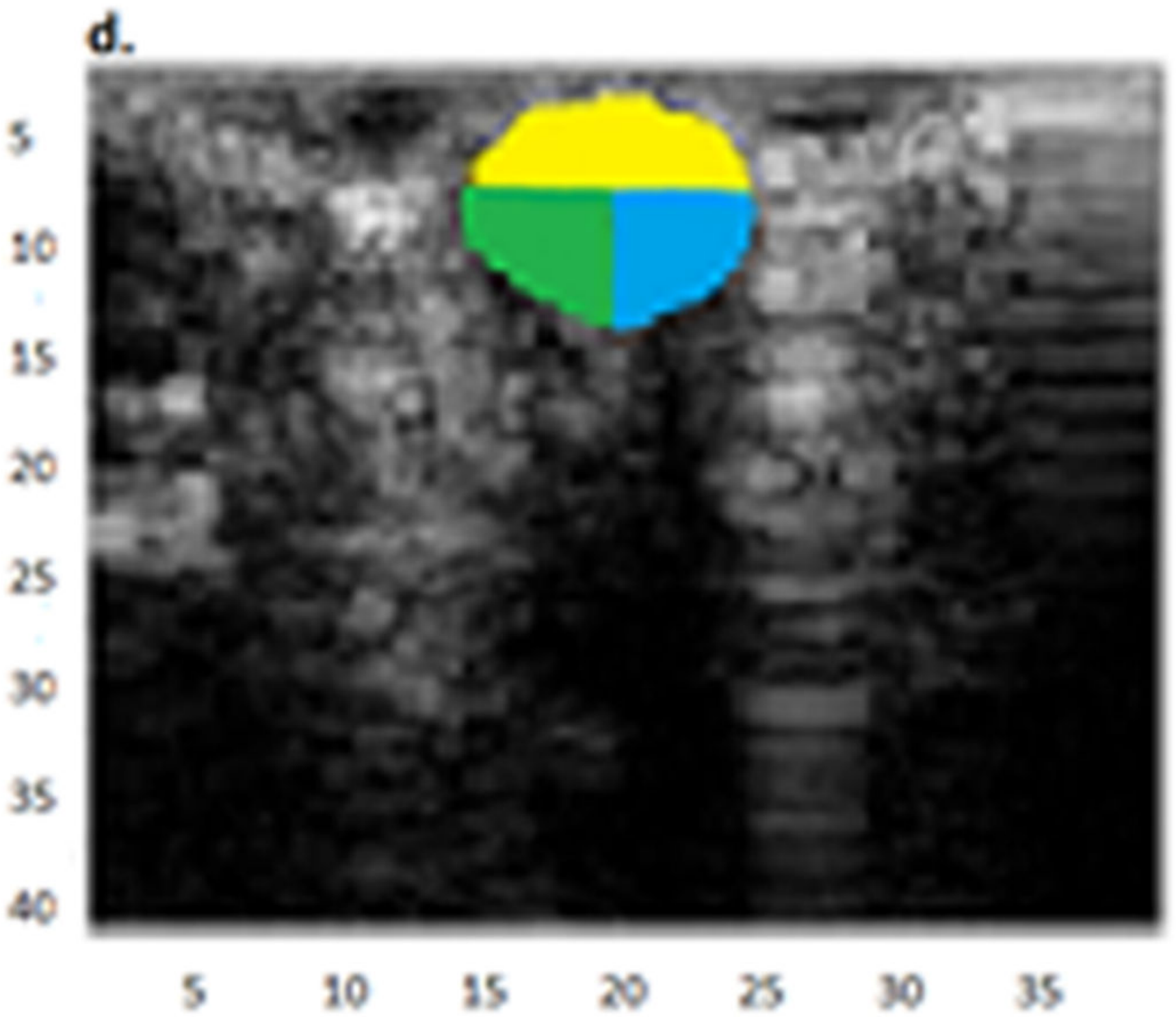

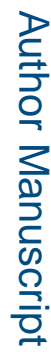




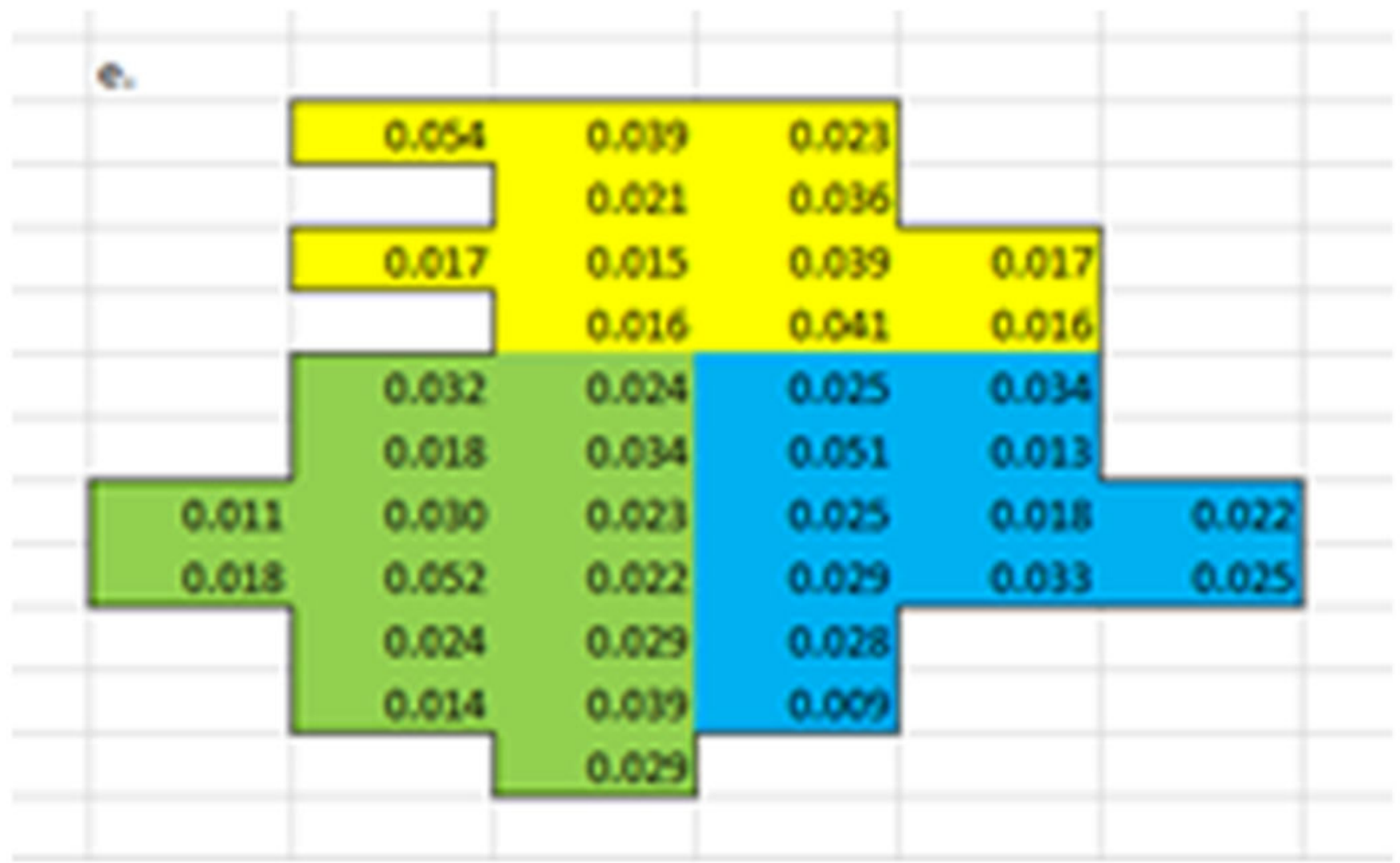




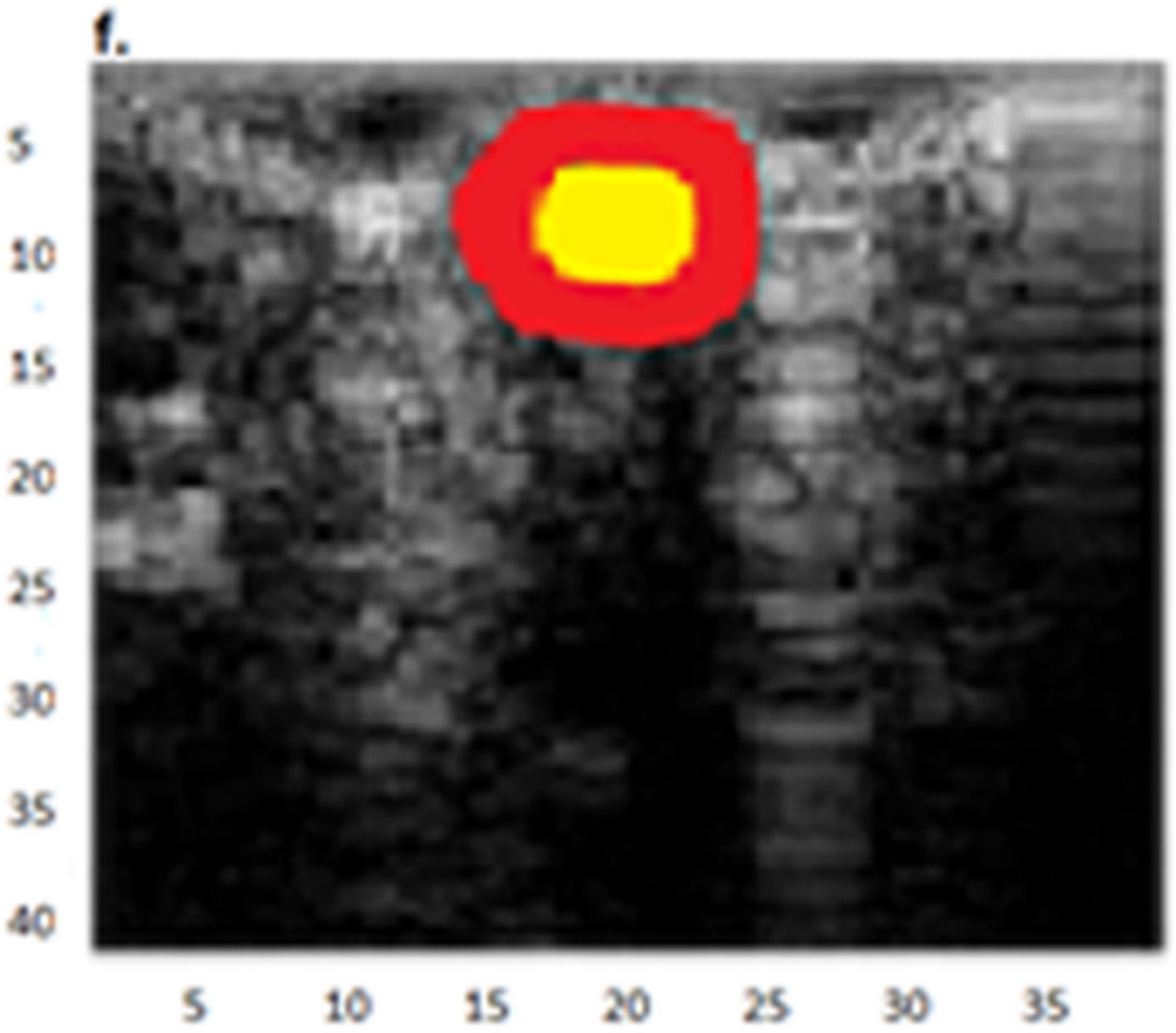

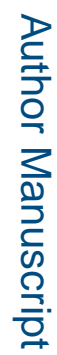




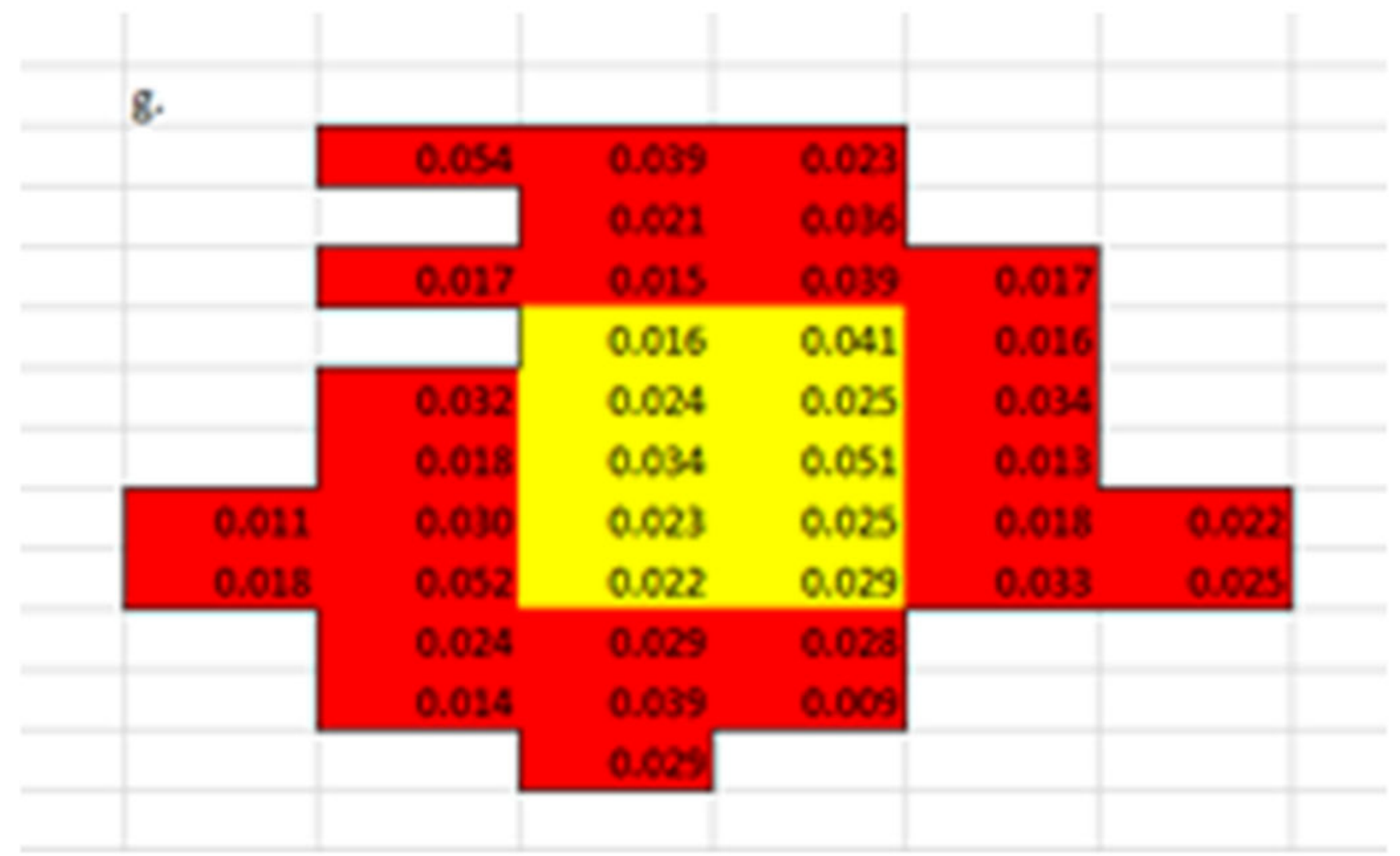




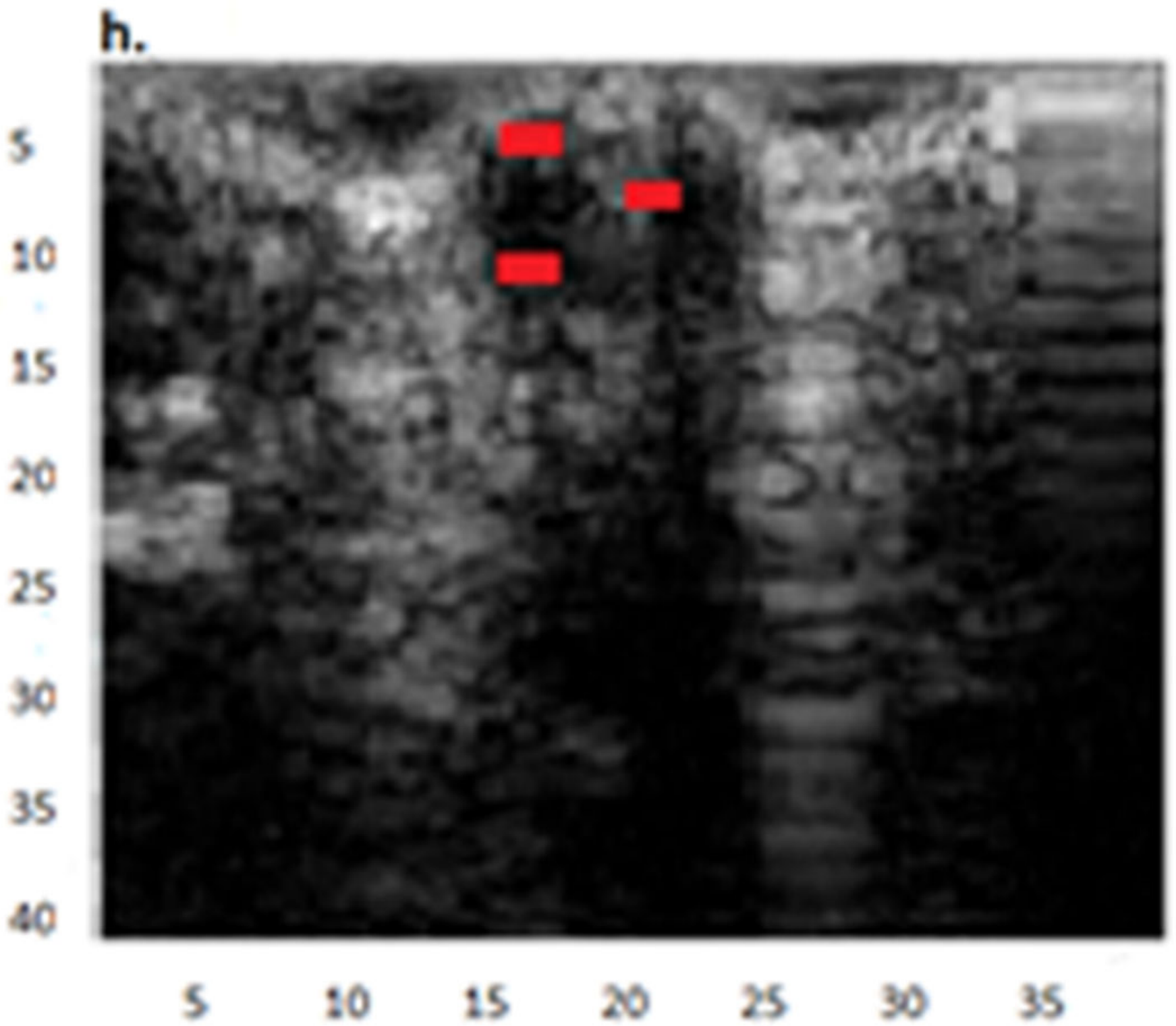

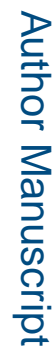




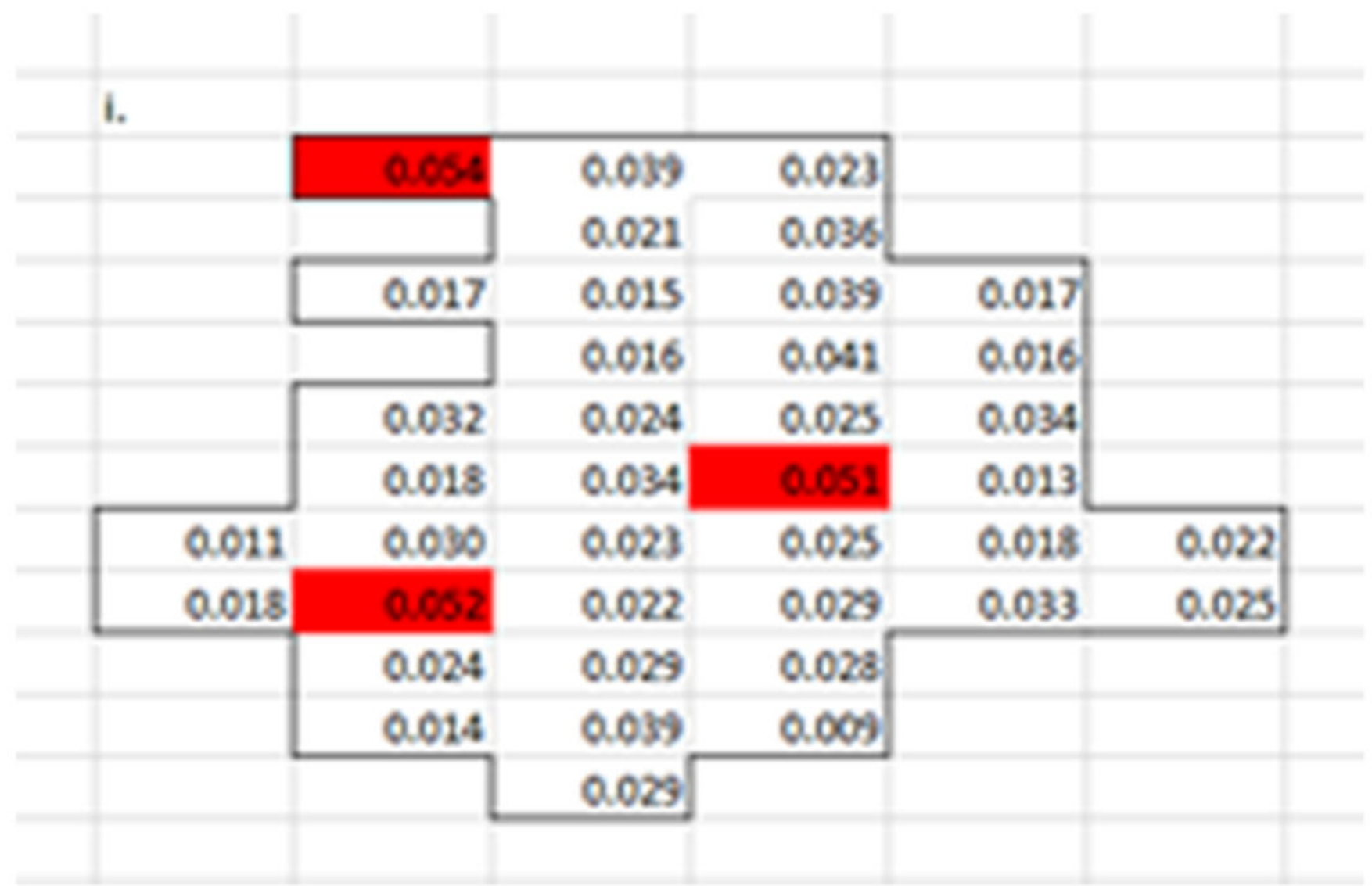

Figure 3:

Tumor (from Figure 1) as it appears in (a) ultrasound scan, (b) with the entire tumor marked as the ROI and (c) the corresponding pathological map for CD31. Also showing (d) the tumor with 3 sub-ROIs marked (yellow, green and blue) and (e) the corresponding pathological map for CD31. Likewise, (f) the scan divided into a central and peripheral ROI (yellow and red, respectively) as well as (g) the corresponding map for CD31. Finally, the matching ultrasound (h) and (i) pathology maps for tumor hotspots is presented. 


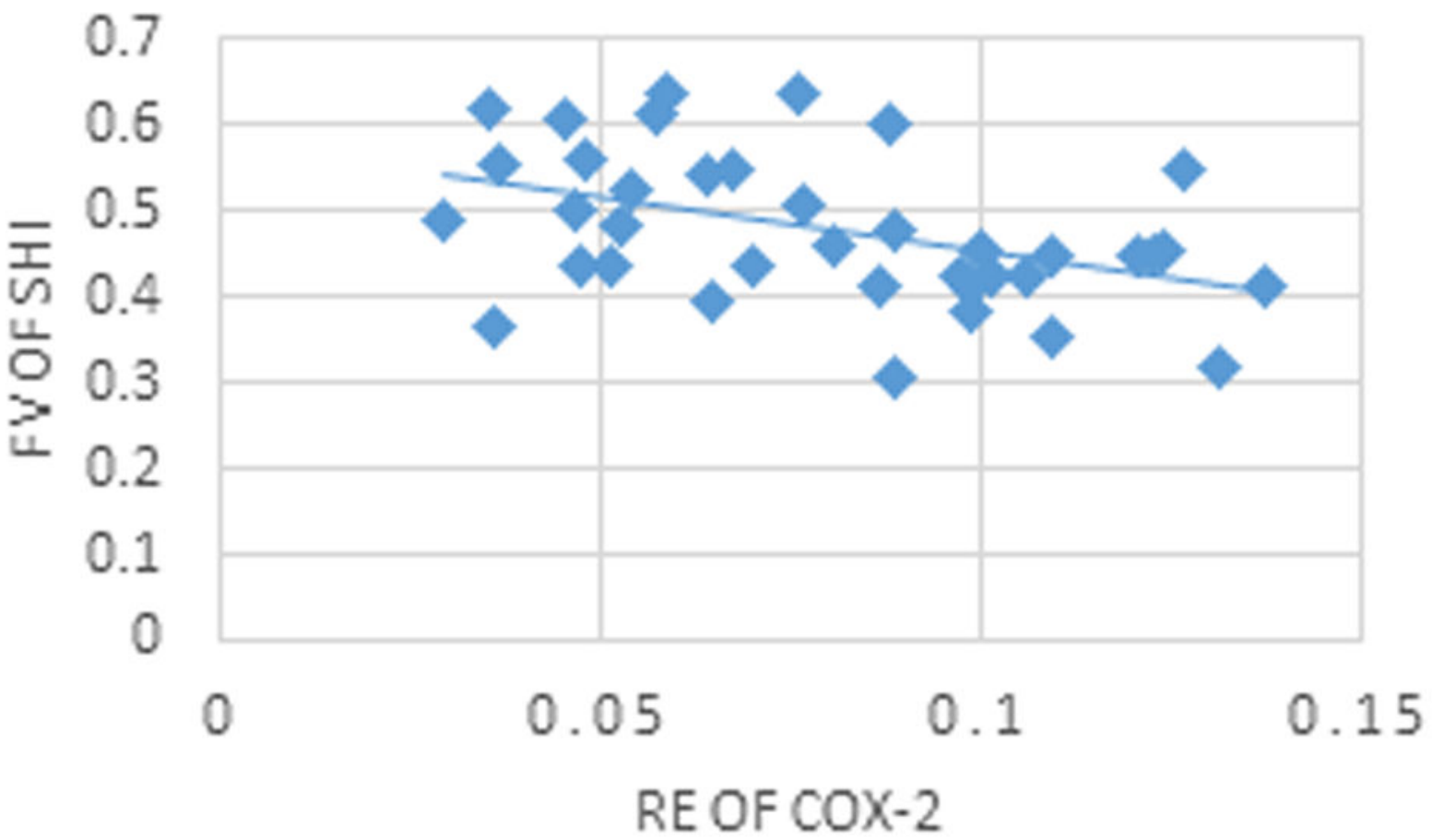




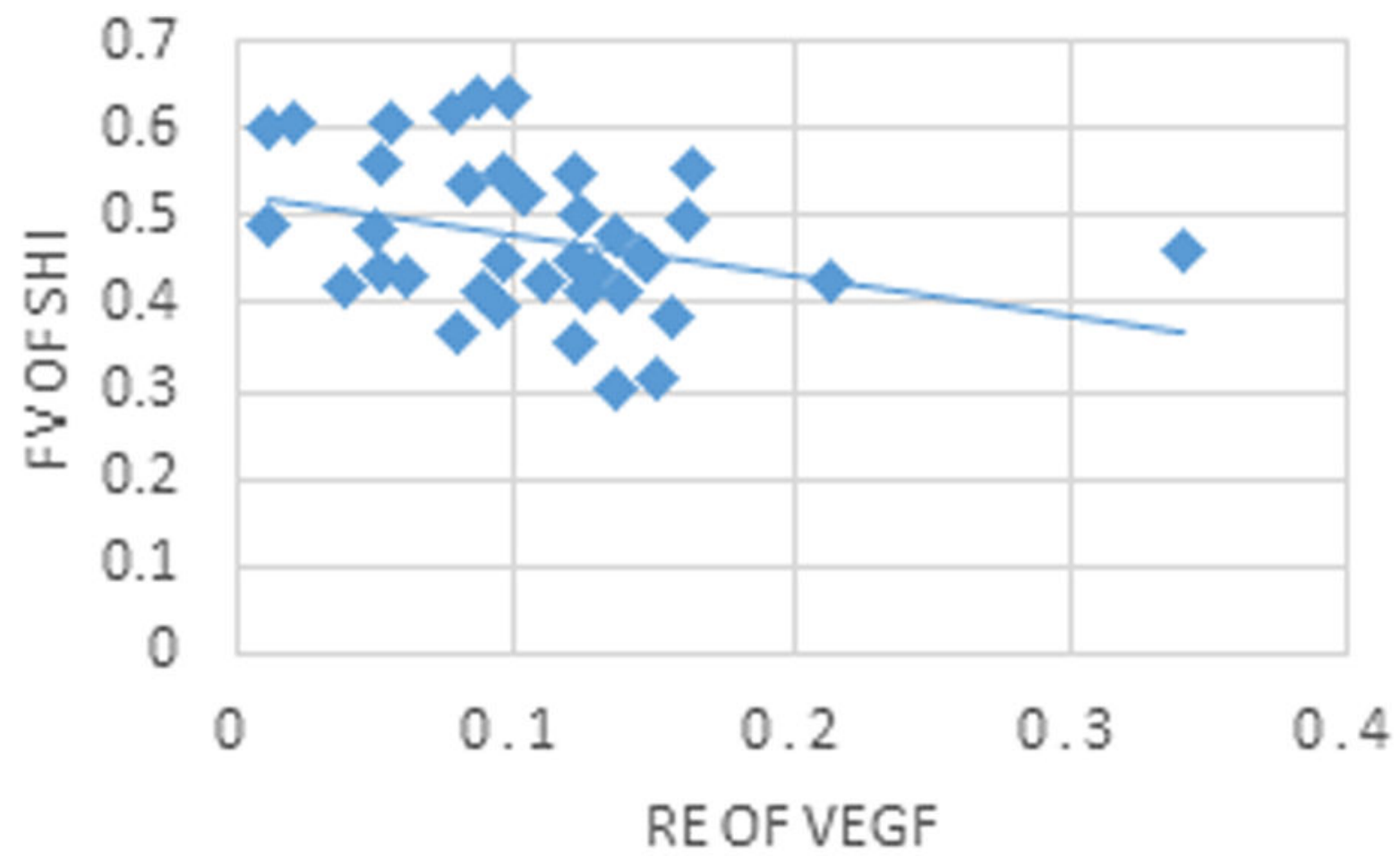

Figure 4:

Scatterplot comparing SHI FVs to REs of (a) COX-2 and (b) VEGF in the tumor periphery. 


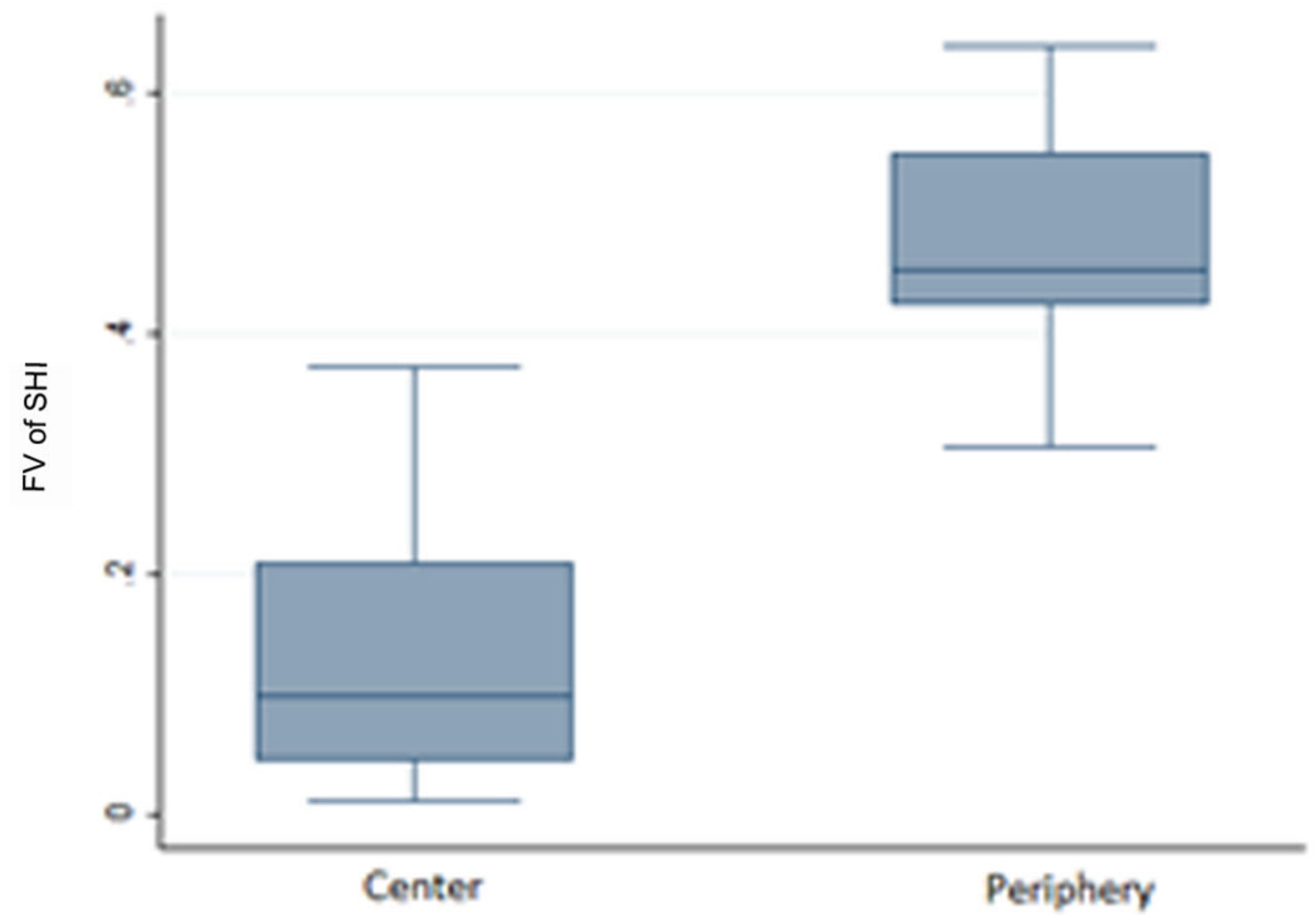




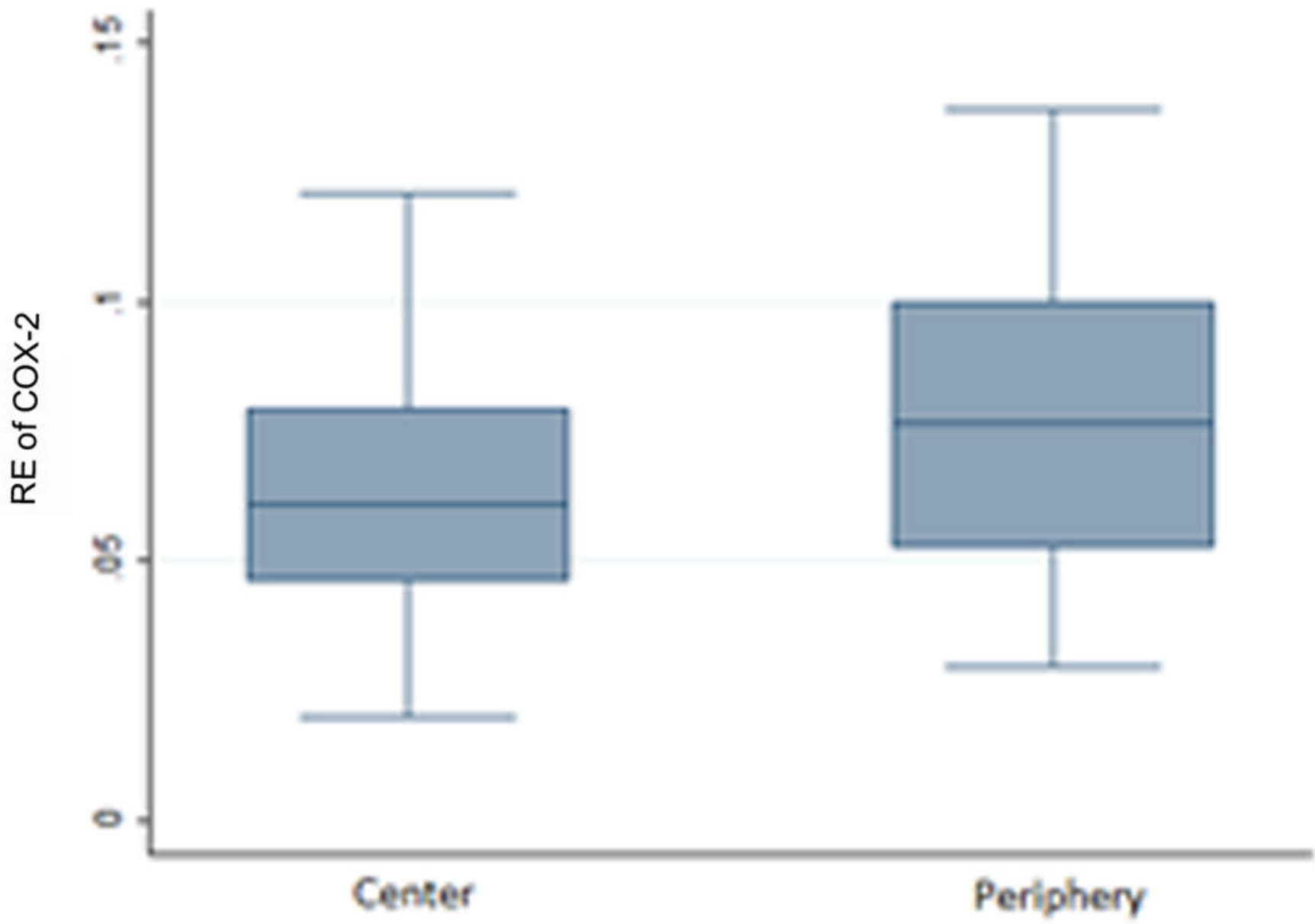

Figure 5.

Box plot of (a) SHI FVs and (b) COX-2 REs when the center vs the periphery of the tumor is considered. 
Table 1:

Image acquisition parameters for SHI on the Sonix RP scanner

\begin{tabular}{cc}
\hline Transducer & L9-4 \\
Mode & Pulse inversion \\
Transmit frequency & $8 \mathrm{MHz}$ \\
Receive frequency & $4 \mathrm{MHz}$ \\
Bandpass filter & $1 \mathrm{MHz}$ bandwidth centered around $4 \mathrm{MHz}$ \\
Power & $-10 \mathrm{~dB}$ \\
Depth & $3 \mathrm{~cm}$ \\
Pulse repetition frequency & $2.56 \mathrm{kHz}$ \\
Sampling frequency & $40 \mathrm{MHz}$ \\
Line density & 128 \\
\hline
\end{tabular}


Table 2:

Linear regression results ( $r$ - and p-values; significant values have been bolded) comparing SHI FVs and REs from the three immunohistochemical markers (CD31, COX-2, VEGF) for the different assessments of neovascularity.

\begin{tabular}{cccccccc}
\hline & \multicolumn{1}{c}{ SHI vs CD31 } & \multicolumn{2}{c}{ SHI vs COX-2 } & \multicolumn{2}{c}{ SHI vs VEGF } \\
& $\mathbf{r}$ & $\mathbf{p}$ & $\mathbf{r}$ & $\mathbf{p}$ & $\mathbf{r}$ & $\mathbf{p}$ \\
\hline Entire tumor $(\mathrm{N}=38)$ & 0.04 & 0.79 & 0.09 & 0.60 & 0.00 & 0.98 \\
Sub-ROIs (N = 105) & -0.13 & 0.18 & 0.01 & 0.89 & -0.07 & 0.47 \\
Centrally (N = 38) & -0.21 & 0.19 & 0.02 & 0.88 & 0.12 & 0.46 \\
Peripherally (N = 38) & 0.21 & 0.21 & -0.42 & $\mathbf{0 . 0 0 5}$ & -0.32 & $\mathbf{0 . 0 4 9}$ \\
Hot spots (N = 114) & 0.11 & 0.23 & 0.25 & $\mathbf{0 . 0 0 7}$ & 0.02 & 0.86 \\
\hline
\end{tabular}

